\title{
Full Scale Bridge Damage Detection Using Sparse Sensor Networks, Principal Component Analysis, and Novelty Detection ${ }^{+}$
}

\author{
Emmanuel Akintunde, Saeed Eftekhar Azam *, Ahmed Rageh and Daniel Linzell \\ Department of Civil Engineering, University of Nebraska-Lincoln, Lincoln, NE 68503, USA; \\ eakintunde@huskers.unl.edu (E.A.); a.eissa.rageh@gmail.com (A.R.); dlinzell@unl.edu (D.L.) \\ * Correspondence: eftekhar-azam@unl.edu \\ + Presented at the 6th International Electronic Conference on Sensors and Applications, \\ 15-30 November 2019; Available online: https://ecsa-6.sciforum.net/.
}

Published: 14 November 2019

\begin{abstract}
Over the decades, visual inspection has been adopted as a means to monitor infrastructure health. While visual inspection provides insights on a bridge's condition, it has been generally agreed that it is insufficient and inefficient. This has called for the creation of autonomous, robust, continuous, and quantitative structural health monitoring (SHM) systems to detect potential deficiencies in an early stage, and monitor future condition. Various methods have been explored that associate changes in condition with changes in the structure's vibration characteristics. These methods have been mostly tested on laboratory specimens experiencing simulated damage. There is need for extending validation of these SHM methods on in-situ structures experiencing real damage under operational and environmental conditions. This paper summarizes a full-scale experiment exploring bridge damage detection effectiveness under variable traffic loads. Three different types of damage were introduced into a full-scale, bridge deck mock-up. These included crash-induced bridge barrier damage, controlled barrier damage, and damage to the deck slab. At the end of each introduced damage case, the bridge's response to the multiple passages was recorded using specific vehicles specifications. Data was extracted and analyzed to identify damage using principal component analysis (PCA) and independent component analysis (ICA) as damagesensitive features. The extracted damage features were thereafter used as input for unsupervised learning (novelty detection). One interesting observation was how PCA revealed possibly significant damage after a crash, which under visual inspection appeared to be minor. Novelty detection using PCA as its damage feature was shown to provide robust damage detection irrespective of load, speed variation, and signal noise levels.
\end{abstract}

Keywords: principal component analysis; damage detection; variable traffic load; strain measurement; crash test; novelty detection; output only

Acknowledgments: The authors would like to acknowledge the support provided by NSF Award \#1762034 BD Spokes: MEDIUM: MIDWEST: Smart Big Data Pipeline for Aging Rural Bridge Transportation Infrastructure (SMARTI). The authors also gratefully acknowledge the assistance, access, computing resources, data, and expertise provided by the University of Nebraska Lincoln's Holland Computing Center, Midwest Roadside Safety Facility staff, and Bridge Diagnostics Inc. in association with this project. Finally, the authors would like to acknowledge assistance by Peter Hilsabeck in conducting the tests.

(C) 2019 by the authors. Licensee MDPI, Basel, Switzerland. This article is an open access article distributed under the terms and conditions of the Creative Commons Attribution (CC BY) license (http://creativecommons.org/licenses/by/4.0/). 